\section{Hämolyse, in vivo und in vitro}

C. Vidal ${ }^{1}$ und W.-R. Külpmann ${ }^{2}$

${ }^{1}$ Landeskriminalamt Niedersachsen, Dezernat 53 „Chemie“, Hannover, Deutschland

${ }^{2}$ Hannover, Deutschland

\section{Englischer Begriff hemolysis}

Definition Auflösung der Erythrozyten.

Beschreibung Hämolyse kann krankheitsbedingt auftreten (In-vivo-Hämolyse) z. B. bei Glukose-6-Phosphatdehydrogenase-Mangel der Erythrozyten, Hämoglobinopathien (Thalassämie, Sichelzellanämie), Wärme- bzw. Kälteantikörpern (Autoimmunerkrankungen), Transfusionszwischenfall, disseminierter intravasaler Gerinnung, Malaria, Vitamin-B ${ }_{12}$, Folsäure- oder Eisenmangel, paroxysmaler nächtlicher Hämoglobinurie. Hämolyse kann bei Probennahme oder -verwahrung entstehen (In-vitroHämolyse): übermäßige Stauung bei Blutentnahme, starkes
Aspirieren, Ausspritzen, Schütteln, Stehenlassen bei Raumtemperatur, Kontamination (Wasser, Detergenzien), hochtouriges Zentrifugieren.

Folgen der Hämolyse:

- Bestandteile, die im Vergleich zum Plasma in hoher Konzentration in Erythrozyten enthalten sind, führen $\mathrm{zu}$ (falsch) hohen Messwerten im Plasma: z. B. Laktatdehydrogenase, Kalium, AST, ALT, Magnesium.

- Hämoglobin in hoher Konzentration stört durch spektrale Interferenz.

- Hämoglobin kann den Reaktionsverlauf von Bestimmungsverfahren stören.

\section{Literatur}

Wisser H (1995) Einflußgrößen und Störgrößen. In: Greiling H, Gressner AM (Hrsg) Lehrbuch der Klinischen Chemie und Pathobiochemie, 3. Aufl. Schattauer Verlag, Stuttgart, S 50-71 